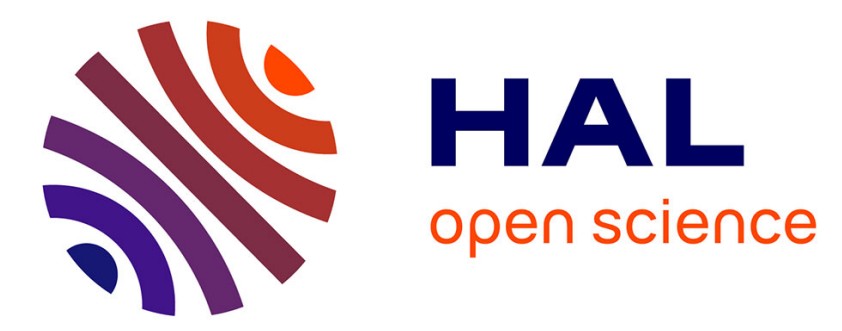

\title{
On the Problem of Choosing the Optimal Parameters for the Wind Farm in the Arctic Town of Tiksi
}

\author{
Sergei Strijhak, Victor Gergel, Aleksandr Ivanov, Sébastien Gadal
}

\section{To cite this version:}

Sergei Strijhak, Victor Gergel, Aleksandr Ivanov, Sébastien Gadal. On the Problem of Choosing the Optimal Parameters for the Wind Farm in the Arctic Town of Tiksi. Balandin D., Barkalov K., Gergel V., Meyerov I. Mathematical Modeling and Supercomputer Technologies, Springer, pp.361-375, 2021, 978-3-030-78758-5. 10.1007/978-3-030-78759-2_30. hal-03274909

\section{HAL Id: hal-03274909 \\ https://hal-amu.archives-ouvertes.fr/hal-03274909}

Submitted on 1 Jul 2021

HAL is a multi-disciplinary open access archive for the deposit and dissemination of scientific research documents, whether they are published or not. The documents may come from teaching and research institutions in France or abroad, or from public or private research centers.
L'archive ouverte pluridisciplinaire HAL, est destinée au dépôt et à la diffusion de documents scientifiques de niveau recherche, publiés ou non, émanant des établissements d'enseignement et de recherche français ou étrangers, des laboratoires publics ou privés.

\section{(1) (1) $\$$}

Distributed under a Creative Commons Attribution - NonCommercial - NoDerivatives 44.0 


\section{Dmitry Balandin}

Konstantin Barkalov

Victor Gergel

Iosif Meyerov (Eds.)

\section{Mathematical Modeling}

and Supercomputer

Technologies

20th International Conference, MMST 2020

Nizhny Novgorod, Russia, November 23-27, 2020

Revised Selected Papers 


\section{Communications in Computer and Information Science}

Editorial Board Members

Joaquim Filipe (1)

Polytechnic Institute of Setúbal, Setúbal, Portugal

Ashish Ghosh

Indian Statistical Institute, Kolkata, India

Raquel Oliveira Prates (1)

Federal University of Minas Gerais (UFMG), Belo Horizonte, Brazil

Lizhu Zhou

Tsinghua University, Beijing, China 
More information about this series at http://www.springer.com/series/7899 
Dmitry Balandin · Konstantin Barkalov • Victor Gergel · Iosif Meyerov (Eds.)

\section{Mathematical Modeling and Supercomputer Technologies}

20th International Conference, MMST 2020

Nizhny Novgorod, Russia, November 23-27, 2020 Revised Selected Papers

黛 Springer 


\section{Editors}

Dmitry Balandin (iD)

Lobachevsky State University of Nizhni

Novgorod

Nizhny Novgorod, Russia

\author{
Victor Gergel (1D \\ Lobachevsky State University of Nizhni \\ Novgorod \\ Nizhny Novgorod, Russia
}

\author{
Konstantin Barkalov (iD \\ Lobachevsky State University of Nizhni \\ Novgorod \\ Nizhny Novgorod, Russia \\ Iosif Meyerov \\ Lobachevsky State University of Nizhni \\ Novgorod \\ Nizhny Novgorod, Russia
}

\author{
ISSN 1865-0929 \\ ISSN 1865-0937 (electronic) \\ Communications in Computer and Information Science \\ ISBN 978-3-030-78758-5 \\ ISBN 978-3-030-78759-2 (eBook)
}

\section{Springer Nature Switzerland AG 2021}

This work is subject to copyright. All rights are reserved by the Publisher, whether the whole or part of the material is concerned, specifically the rights of translation, reprinting, reuse of illustrations, recitation, broadcasting, reproduction on microfilms or in any other physical way, and transmission or information storage and retrieval, electronic adaptation, computer software, or by similar or dissimilar methodology now known or hereafter developed.

The use of general descriptive names, registered names, trademarks, service marks, etc. in this publication does not imply, even in the absence of a specific statement, that such names are exempt from the relevant protective laws and regulations and therefore free for general use.

The publisher, the authors and the editors are safe to assume that the advice and information in this book are believed to be true and accurate at the date of publication. Neither the publisher nor the authors or the editors give a warranty, expressed or implied, with respect to the material contained herein or for any errors or omissions that may have been made. The publisher remains neutral with regard to jurisdictional claims in published maps and institutional affiliations.

This Springer imprint is published by the registered company Springer Nature Switzerland AG The registered company address is: Gewerbestrasse 11, 6330 Cham, Switzerland 


\section{Preface}

The 20th International Conference and School for Young Scientists "Mathematical Modeling and Supercomputer Technologies" (MMST 2020) was held during November 23-27, 2020, in Nizhni Novgorod, Russia. The conference and school were organized by the Mathematical Center "Mathematics of Future Technologies" and the Research and Educational Center for Supercomputer Technologies of the Lobachevsky State University of Nizhni Novgorod. The conference was supported by reputable sponsors (Intel, Huawei, and RSC). It was organized in partnership with the International Congress "Russian Supercomputing Days".

The topics of the conference and school covered a wide range of problems related to mathematical modeling of complex processes and numerical methods of research, as well as new methods of supercomputing aimed at solving state-of-the-art problems in various fields of science, industry, business, and education.

This edition of the MMST conference was dedicated to the 100th birthday of Professor Yuri Neimark. Professor Neimark's contribution to science and higher education is truly immense. He has left considerable heritage in the qualitative theory of differential equations, the theory of stability, the theory of adaptive and robust control, pattern recognition, nonholonomic and gyroscopic systems mechanics, optimization, and mathematical modeling. Yuri Neimark founded the USSR's first Faculty of Computational Mathematics and Cybernetics and the Research Institute of Applied Mathematics and Cybernetics. He is the author of about 600 published works, including at least 12 for inventions and 10 monographs.

The scientific program of the conference featured the following plenary lectures given by outstanding scientists:

- Alexander Boukhanovsky (Russia): Generative design of value-based systems.

- Vladimir Voevodin (Russia): AlgoWiki, the structure of algorithms and the impossible becomes possible.

- Alexander Gorban (UK): The new centaur: man and artificial intelligence.

- Andrei Gritsun (Russia): Predicting Earth climate change using the INM RAS Earth system model.

- Aleksey Eliseev (Russia): The IAP RAS Earth system model: state of the art and review of key findings.

- Aleksey Koronovskii (Russia): Multistability in an intermittent generalized chaotic synchronization regime.

- Evgeniy Mareev (Russia): Current problems in geophysical electrodynamics.

- Alexander Moskovsky (Russia): Unique and effective RSC solutions for complex problems.

- Sergey Pavlov (Russia): ARM Ecosystem Development for AI, Cloud and High Performance Computing.

- Arkady Pikovsky (Germany): Low-dimensional reduction for ensembles of noisy oscillators. 


\title{
On the Problem of Choosing the Optimal Parameters for the Wind Farm in the Arctic Town of Tiksi
}

\author{
Sergei V. Strijhak ${ }^{1,2}\left(\mathbb{D}\right.$, Victor P. Gergel ${ }^{3}\left(\mathbb{0}\right.$, Aleksandr V. Ivanov $^{1(\bowtie)}(\mathbb{D}$, \\ and Sebastien Zh. Gadal ${ }^{4,5}$
}

1 Ivannikov Institute for System Programming of the RAS, 109004 Moscow, Russia

\{s.strijhak, av.ivanov\}@ispras.ru

2 Moscow Aviation Institute, 125993 Moscow, Russia

3 Lobachevsky State University of Nizhny Novgorod, 603950 Nizhnij Novgorod, Russia gergel@unn.ru

4 Aix-Marseille Univ, CNRS, ESPACE UMR 7300, Univ Nice Sophia Antipolis, Avignon Univ, 13545 Aix-en-Provence, France sebastien.gadal@univ-amu.fr

5 North-Eastern Federal University, 67000 Yakutsk, Republic of Sakha Yakutia, Russia

\begin{abstract}
The paper considers the problem of choosing the optimal parameters for the operation of 3 horizontal wind turbines of the wind farm in the town Tiksi, in the Sakha Republic. The open-source WRFARW and FLORIS packages are used to calculate the physical parameters in the wind farm. During the calculation, the values of wind velocity, temperature, pressure fields, and the value of the generated power of wind power plants were obtained. The optimization problem is formulated, the objective function is defined, and 2 variable parameters are selected, namely, yaw angle and angle of attack. The calculations were carried out on the computing cluster of ISP RAS.
\end{abstract}

Keywords: Wind turbine $\cdot$ Wind farm $\cdot$ Calculation $\cdot$ Domain $\cdot$ Grid · Wind velocity $\cdot$ Objective function · Wind turbine parameters • Yaw angle $\cdot$ Power

\section{Introduction}

The design and selection of optimal parameters for horizontal wind turbines in the wind farm is an urgent task, especially in the case of the construction of new wind farms in the Russian Federation. One of the interesting areas for research may be the problem of choosing the best operating modes of the wind farm, taking into account the influence of local weather conditions, as well as the relative location of the wind turbine in a real area. The variable parameters include the angular rotation velocity of the wind turbine, the yaw angle, the angle of rotation of the blade with respect to the vector of the incoming flow, and others. 


\section{Wind Farm in the Town Tiksi}

The wind farm built by RusHydro together with its Japanese partners in the Arctic town Tiksi, which is home to more than 4.6 thousand people, has demonstrated high efficiency and reliability in the harsh Arctic climate. In the winter of 2018-2020, the air temperature in Tiksi dropped to minus $42^{\circ}$, and the wind velocity reached $30 \mathrm{~m} / \mathrm{s}$.

In November 2018, in the Arctic urban-type settlement of Tiksi, which is an important transport hub for the Northern Sea Route, 3 wind turbines from Komaihaltec company from Japan with a capacity of $0.3 \mathrm{MW}$ each were put into operation with the participation of Sakhaenergo and RusHydro. Now the wind farm works together with the existing diesel power plant of the town.

The wind turbines (WT) of the Tiksi wind farm are located at an altitude of about $120 \mathrm{~m}$ above sea level, $4 \mathrm{~km}$ from the town Tiksi and $2.7 \mathrm{~km}$ from the Laptev Sea, Fig. 1.

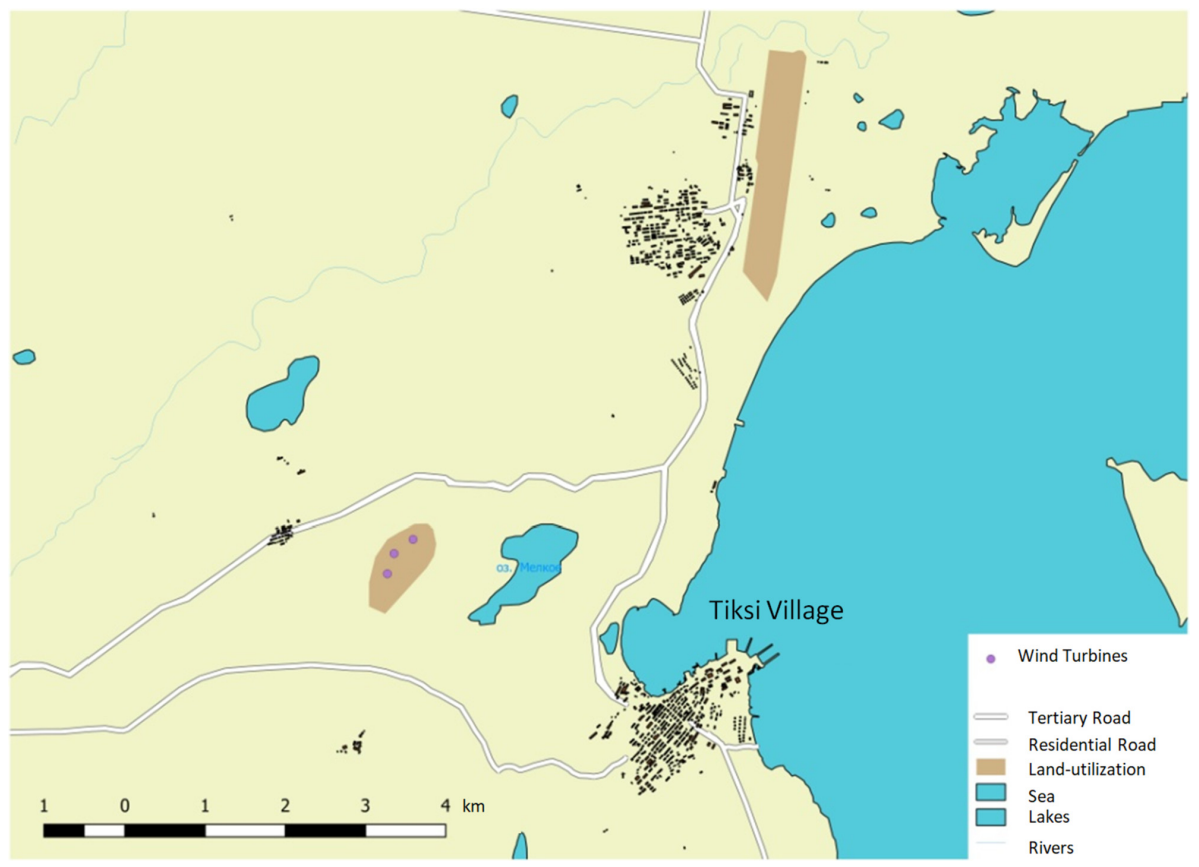

Fig. 1. The map of the town in Tiksi. Open Street Map, 11.09.2019.

Turbines coordinates are: $71^{\circ} 39^{\prime} 24.8^{\prime \prime} \mathrm{N} 128^{\circ} 46^{\prime} 17.6^{\prime \prime} \mathrm{E}$ (WT1), $71^{\circ} 39^{\prime} 20 .^{\prime \prime} \mathrm{N}$ $128^{\circ} 45^{\prime} 55.7^{\prime \prime} \mathrm{E}$ (WT2), $71^{\circ} 39^{\prime} 12.02^{\prime \prime} \mathrm{N} 128^{\circ} 45^{\prime} 43.57^{\prime \prime} \mathrm{E}$ (WT3), see Fig. 2. 


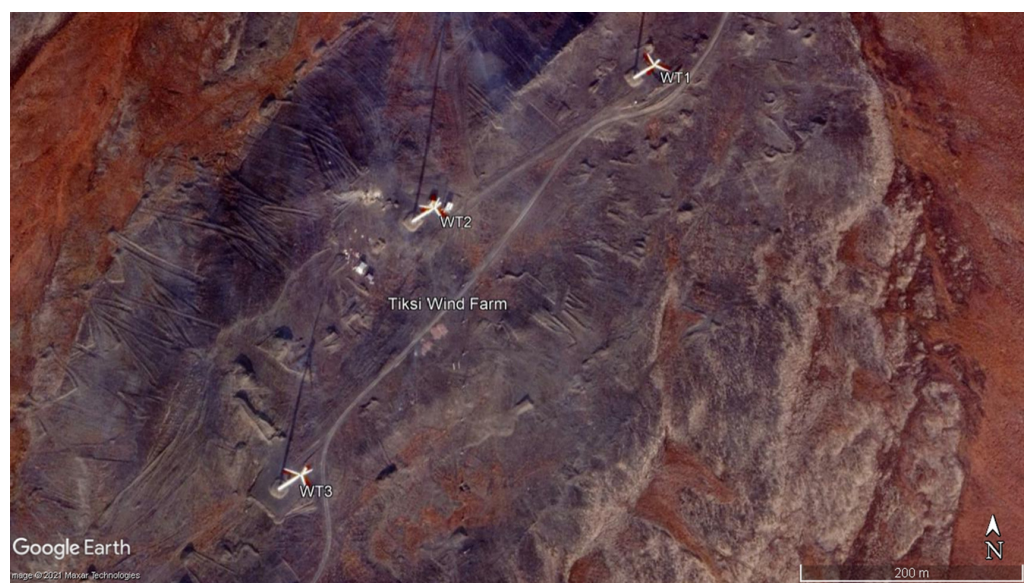

Fig. 2. Satellite image of the wind turbines location. Google Earth, 25.09.2020.

\subsection{Mathematical Model in WRF-ARW Code}

The calculation code WRF-ARW (WRF - Weather Research and Forecasting, ARW - Advanced Research WRF) is actively used to predict the capacity of the wind farm. The WRF-ARW model is a model of a regional weather forecast, for the construction of which it needs boundary and initial conditions [1,2]. Data sources in this case can be global models, observational data, or forecast reanalysis data. As a parent model for WRF-ARW, data from calculations based on the Global Forecast System (GFS) model is usually used. Figure 3 shows an algorithm for the interaction of different calculation models for different levels of calculation of physical quantities.

The WRF-ARW model allows one to obtain a large range of physical quantities that describe weather conditions. As a rule, the main parameters are the distribution of wind velocity, temperature, and pressure. Secondary parameters may include air humidity, precipitation distribution, precipitation type, and much more. In WRF-ARW, there are various parameterization models, including the model for parameterizing the operation of a wind turbine - the Actuator Disk Model [3].

The WRF-ARW model is based on non-hydrostatic equations for a compressible fluid written in Cartesian coordinates horizontally and using the orographic coordinate $\eta$ vertically. In older versions of the package, $\eta$ was determined using the hydrostatic pressure $p_{h}$ :

$$
\eta=\frac{p_{d}-p_{t}}{p_{s}-p_{t}}
$$

where $p_{d}$ is the hydrostatic component of dry air pressure, $p_{s}$ and $p_{h}$ refer to values of $p_{d}$ along the surface and top boundaries respectively. In WRF-ARW 
version 4, the vertical coordinate was generalized to reduce the influence of the surface on the coordinate grid with increasing height:

$$
p_{d}=B(\eta)\left(p_{s}-p_{h}\right)+[\eta-B(\eta)]\left(p_{0}-p_{h}\right)+p_{h},
$$

where $p_{0}$ is a reference sea-level pressure. Here $B(\eta)$ defines the relative weighting between the terrain-following sigma coordinate and a pure pressure coordinate, such that $\eta$ corresponds to the sigma coordinate (1) for $B(\eta)=\eta$ and reverts to a hydrostatic pressure coordinate for $B(\eta)=0$. For a smooth transition from the sigma coordinate near the surface to the pressure coordinate at the upper levels, $B(\eta)$ is defined by a third order polynomial.

The governing equations system is represented as follows. The momentum equations are written as

$$
\begin{aligned}
\partial_{t} U & +m_{x}\left[\partial_{x}(U u)+\partial_{y}(V u)\right]+\partial_{\eta}(\Omega u) \\
& +\left(m_{x} / m_{y}\right)\left(\alpha / \alpha_{d}\right)\left[\mu_{d}\left(\partial_{x} \phi^{\prime}+\alpha_{d} \partial_{x} p^{\prime}+\alpha_{d}^{\prime} \partial_{x} \bar{p}\right)+\partial_{x} \phi\left(\partial_{\eta} p^{\prime}-\mu_{d}^{\prime}\right)\right]=F_{U} \\
\partial_{t} V & +m_{y}\left[\partial_{x}(U v)+\partial_{y}(V v)\right]+\left(m_{y} / m_{x}\right) \partial_{\eta}(\Omega u) \\
& +\left(m_{y} / m_{x}\right)\left(\alpha / \alpha_{d}\right)\left[\mu_{d}\left(\partial_{y} \phi^{\prime}+\alpha_{d} \partial_{y} p^{\prime}+\alpha_{d}^{\prime} \partial_{y} \bar{p}\right)+\partial_{y} \phi\left(\partial_{\eta} p^{\prime}-\mu_{d}^{\prime}\right)\right]=F_{V} \\
\partial_{t} W & +m_{x}\left[\partial_{x}(U w)+\partial_{y}(V w)\right]+\partial_{\eta}(\Omega w) \\
& -m_{y}^{-1} g\left(\alpha / \alpha_{d}\right)\left[\partial_{\eta} p^{\prime}-\bar{\mu}_{d}\left(q_{v}+q_{c}+q_{r}\right)\right]+m_{y}^{-1} \mu_{d}^{\prime} g=F_{W}
\end{aligned}
$$

and the mass conservation equation and geopotential equation is given by

$$
\begin{aligned}
& \partial_{t} \mu_{d}^{\prime}+m_{x} m_{y}\left[\partial_{x} U+\partial_{y} V\right]+m_{y} \partial_{\eta} \Omega=0, \\
& \partial_{t} \phi^{\prime}+\mu_{d}^{-1}\left[m_{x} m_{y}\left(U \partial_{x} \phi+V \partial_{y} \phi\right)+m_{y} \Omega \partial_{\eta} \phi-m_{y} g W\right]=0 .
\end{aligned}
$$

The conservation equations for the potential temperature $\Theta_{m}$ and the scalar moisture $Q_{m}$ equations:

$$
\begin{aligned}
& \partial_{t} \Theta_{m}+m_{x} m_{y}\left[\partial_{x}\left(U \theta_{m}\right)+\partial_{y}\left(V \theta_{m}\right)\right]+m_{y} \partial_{\eta}\left(\Omega \theta_{m}\right)=F_{\Theta_{m}} \\
& \partial_{t} Q_{m}+m_{x} m_{y}\left[\partial_{x}\left(U q_{m}\right)+\partial_{y}\left(V q_{m}\right)\right]+m_{y} \partial_{\eta}\left(\Omega q_{m}\right)=F_{Q_{m}}
\end{aligned}
$$

and the diagnostic equation for dry hydrostatic pressure:

$$
\partial_{\eta} \phi^{\prime}=-\bar{\mu}_{d} \alpha_{d}^{\prime}-\alpha_{d} \mu_{d}^{\prime}
$$

with the diagnostic relation for the full pressure (dry air plus water vapor):

$$
p=p_{0}\left(\frac{R_{d} \theta_{m}}{p_{0} \alpha_{d}}\right)^{\gamma} .
$$

The following notations is used in the system (2)-(10):

$$
\begin{gathered}
U=\mu_{d} u / m_{y}, \quad V=\mu_{d} v / m_{x}, \quad W=\mu_{d} w / m_{y}, \quad \Omega=\mu_{d} \omega / m_{y}, \\
\Theta_{m}=\mu_{d} \theta_{m}, \quad Q_{m}=\mu_{d} q_{m},
\end{gathered}
$$


where $\mathbf{v}=(u, v, w)$ - velocity vector, $\omega=\dot{\eta}$ - vertical velocity in terms of orographic coordinates, $\mu_{d}$ defines mass of the dry air column, $\phi$ - geopotential, $m_{x}, m_{y}-$ map scale factors. $\theta_{m}=\theta\left(1+\left(R_{v} / R_{d}\right) q_{v}\right) \approx \theta\left(1+1.61 q_{v}\right)$ is the moist potential temperature and $q_{m}=q_{v}, q_{c}, q_{r}, q_{i} \ldots$ represents the mixing ratios of moisture variables (water vapor, cloud water, rain water, ...). $\alpha_{d}$ is the inverse density of the dry air $\left(1 / \rho_{d}\right)$ and $\alpha$ is the inverse density taking into account the full parcel density $\alpha=\alpha_{d}\left(1+q_{v}+q_{c}+q_{r}+q_{i}+\ldots\right)^{-1}$. The right-hand-side terms $F_{U}, F_{V}, F_{W}, F_{\Theta_{m}}$ and $F_{Q_{m}}$ represent forcing terms arising from physical models, turbulent mixing, spherical projections, and the earth's rotation, $g$ - acceleration due to the gravity. Hydrostatically-balanced reference state variables (denoted by overbars) are a function of height only and satisfy the governing equations for an atmosphere at rest.

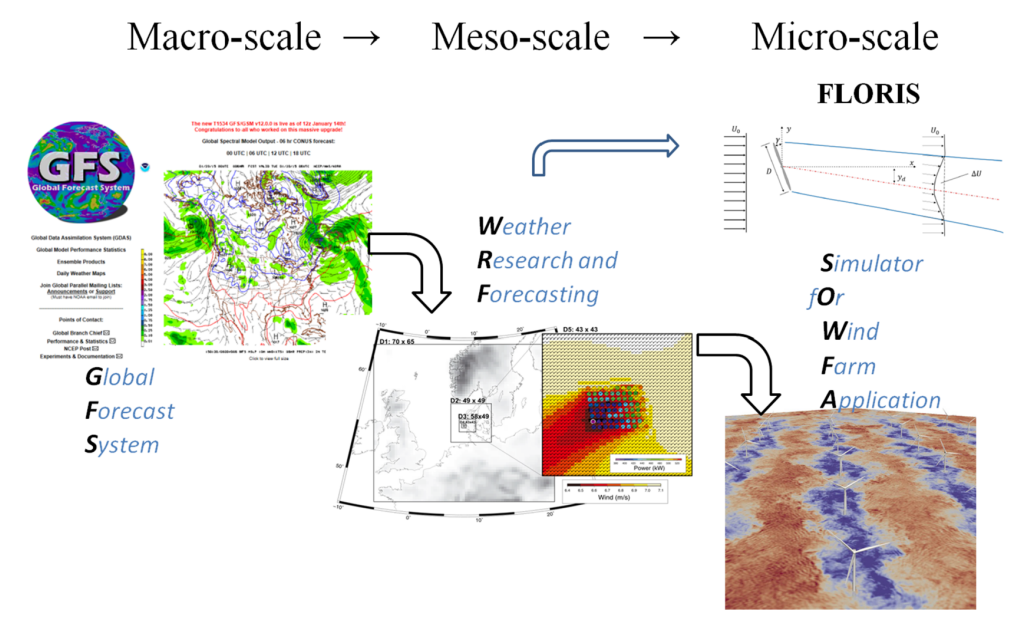

Fig. 3. How to model the operation of a wind farm.

WRF-ARW code supports the configuration of the shared and distributed memory of server. The code could be compiled with OpenMP and OpenMPI libraries for parallel calculations. The goal of parallel option is to speed-up the computing time on supercomputer.

\subsection{Numerical Domain, Grid, and Results of Calculation}

The layout of the numerical domain for the area of the town Tiksi with the image of nested domains is shown in Fig. 4. The calculations in the WRF-ARW package were performed for the following calendar dates: 29.09.2019, 30.09.2019, 01.10.2019. 


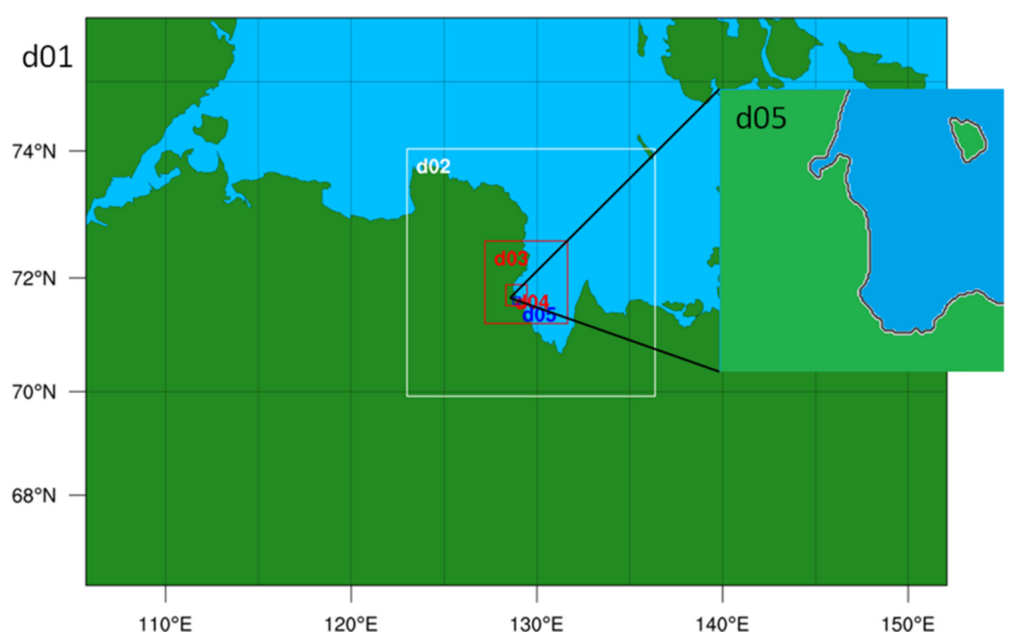

Fig. 4. Layout of the main area with the image of nested domains.

To compare the results, the Tiksi weather station was selected: $71^{\circ} 34^{\prime} 48^{\prime \prime}$ $\mathrm{N} 128^{\circ} 54^{\prime} \mathrm{E}$, altitude $7 \mathrm{~m}$ above sea level. Data on the temperature at the level of $2 \mathrm{~m}$ above the surface, the pressure, and wind velocity at the surface (at the level of the weather station) were displayed at the point closest to the location of the weather station in the d05 region. The graph of the velocity comparison is shown in Fig. 6. The model configuration and simulation results are given in [4].

In general, the results of the model correspond to real data, in any case, they repeat the profile of changes in indicators. Also, the wind farm energy generation was modeled using the built-in model, which was analyzed in [3]. The characteristics of the wind turbine, namely: the power curve and the thrust coefficient as a function of velocity, were taken from the parameters of similar wind turbines from open sources on the Internet (Fig. 5). The result of the simulation is the dynamics of the power of the Tiksi wind farm in a given period, Fig. 7. One can see that the results correspond to the average wind value in the specified area.

The results of the calculation showed that the efficiency of the wind farm does not exceed $20 \%$ for the selected days. In this regard, there is a need to choose the optimal parameters for the operation of wind turbines. This parameter can be the angle of rotation of the nacelle (the yaw angle).

The calculations were performed using 12-cores computer node. Several cases with different configuration of physical parameters were launched simultaneously. The average computation time for one case was about $24 \mathrm{~h}$. 


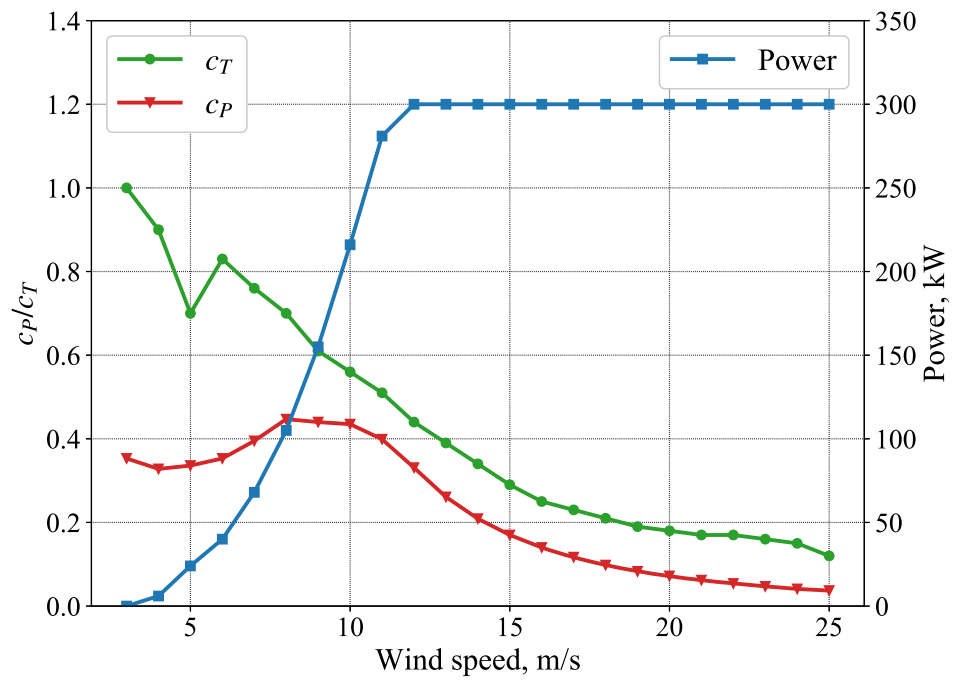

Fig. 5. The power curve and $c_{P}, c_{T}$ coefficients for a single wind turbine.

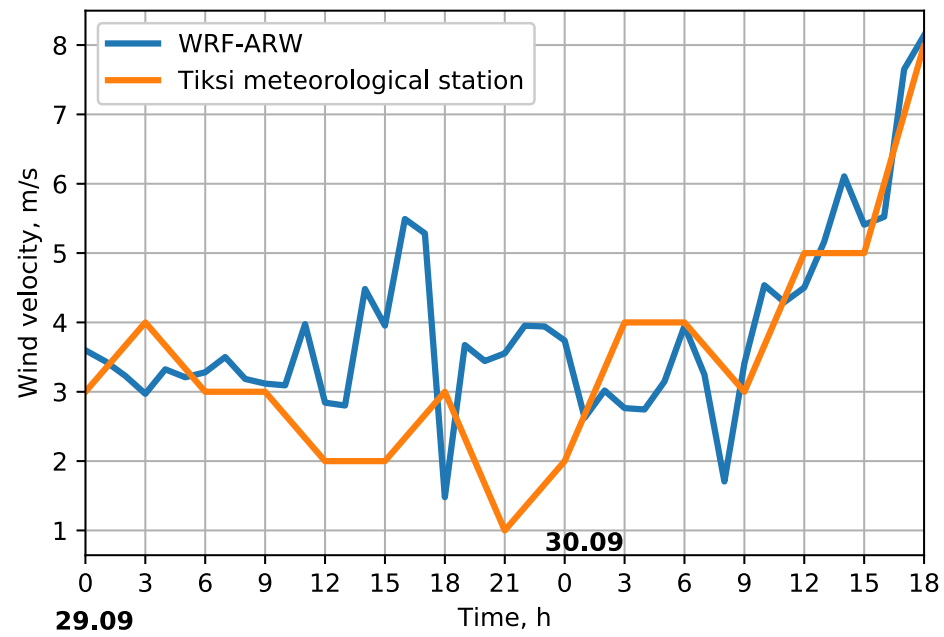

Fig. 6. Comparison of wind velocity at the location of the Tiksi weather station for model and real data. 


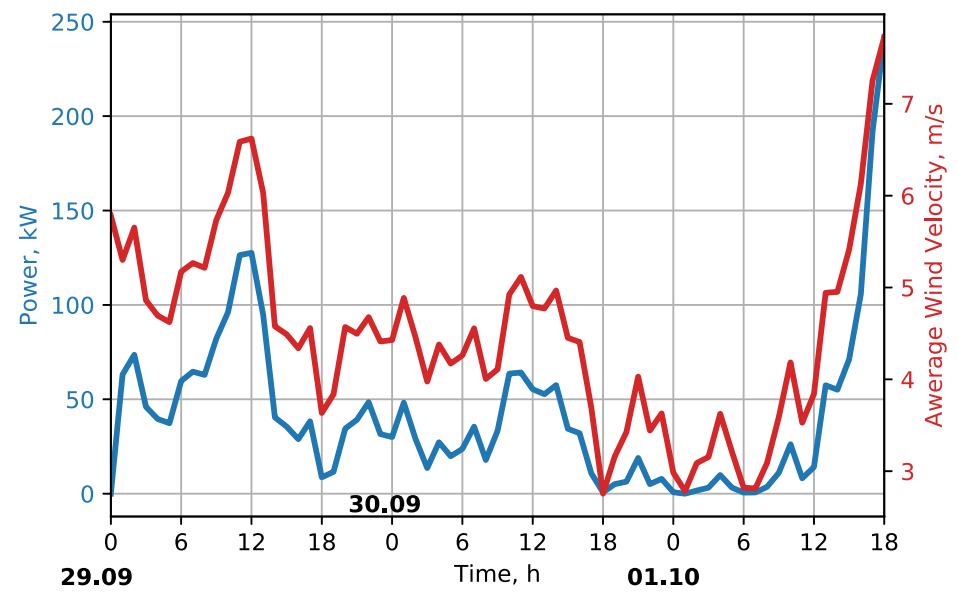

Fig. 7. Simulation change in the total power generation capacity of the Tiksi wind farm on 29.09-01.10.

The simulations were done using the resources of the UniHUB HPC cluster of ISP RAS with 32 computer nodes.

\subsection{Setting Task of Optimizing the Generated Capacity of the Wind Farm}

It is known that the front wind turbines have a significant impact on the operation of the rear wind turbines. There is a concept of velocity deficit. The vortex trail behind the wind turbine may deviate.

Data from a weather station located $10 \mathrm{~km}$ from the wind farm shows that the wind direction changes in the range of angles from $\theta_{1}=0^{\circ}$ to $\theta_{2}=90^{\circ}$. Thus, there is the problem of choosing the optimal angle of rotation of the engine nacelle of the wind turbine (yaw rate), the angle of rotation of the blade to obtain maximum power capacity of wind turbines.

Work in the field of optimization calculations for wind farms and the design of wind turbines was practically not carried out earlier in the Russian Federation. Among the international teams that deal with optimization issues in the design of new wind farms, there are research groups from JHU, USA (Prof. C. Meneveau); KU Leuven, Belgium (Dr. Johan Meyers); EPFL, Lausanne, Switzerland (Prof. Fernando Porte-Agel); NREL (Dr. P. A. Fleming); Stanford University (Dr. J. Park); Aalborg University, Aalborg, Denmark (Prof. Zheng Chen) [5-14]. These teams considered the following tasks:

1. optimization of the location of wind turbines in the wind farm [5-7];

2. selection of optimal parameters (yaw angle of the wind wheel axis, angular rotation velocity, control parameters, etc.) [10-14]; 
3. optimization of the geometric shape of the wind turbine blade in order to reduce the noise level on the ground and increase the durability of the structure made of composite material.

To solve such problems, an objective function was formed, which took into account various criteria and restrictions on the parameters to be changed. Among the optimization methods used, one can distinguish:

- genetic algorithms;

- sequential quadratic programming;

- cross-entropy method;

- an approach based on a combination of the conjugate gradient method, the Polak-Ribière method for determining weight coefficients, and the Brent search algorithm $[8,9]$;

- machine learning methods based on "Bayesian ascent" [10,11];

- the particle swarm method $[12,13]$.

The yaw angle's optimization problem for the wind wheel axis was studied in papers [15-17].

The authors of the work [9] solved the optimization problem using an approach with a cooperative game. All agents (participants) strive to maximize the overall goal. Interaction between agents is taken into account.

The vector maximization problem is solved:

$$
\begin{gathered}
\boldsymbol{x}^{*}=\arg \max _{\boldsymbol{x}} \sum_{i=1}^{N} \varphi_{i} f_{i}(\boldsymbol{x}), \quad \varphi_{i}=1, i=\overline{1, N} \\
\underset{\boldsymbol{x}}{\operatorname{maximize}} f(\boldsymbol{x}) \triangleq \sum_{i=1}^{N} P_{i}\left(\boldsymbol{\alpha}, \boldsymbol{o}, U, \theta^{W}\right), \quad \text { subject to } \boldsymbol{x}^{l} \leqslant \boldsymbol{x} \leqslant \boldsymbol{x}^{u},
\end{gathered}
$$

where $\boldsymbol{x}^{l}, \boldsymbol{x}^{u}$ - lower and upper bounds, $\varphi_{i}$ represents the weighting coefficient on the objective function $f_{i}(x)$.

Because it is difficult to accurately determine the analytical function for the cardinality, the authors used Bayesian optimization (BO).

The problem is solved:

$$
\boldsymbol{x}^{*}=\arg \max _{\boldsymbol{x}} f(\boldsymbol{x})
$$

Observing for the behavior of the function:

$$
y=f(\boldsymbol{x})+\epsilon .
$$

The BO uses 2 phases: training and optimization.

In this paper, the objective function $f(\boldsymbol{x})$ associated with the total power generation was formulated.

It is known that the power for a single wind turbine is equal to:

$$
P=\frac{1}{2} \rho A U^{3} C_{p}(\alpha, 0) .
$$


An arbitrary set of parameters can be considered in an optimization problem:

$$
\boldsymbol{x}=\left(x_{1}, \ldots, x_{i}, \ldots, x_{N}\right) .
$$

The target function for the total capacity of the wind farm is defined as:

$$
f_{i}(\boldsymbol{x})=P_{i}\left(\boldsymbol{\alpha}, \boldsymbol{o}, U, \theta^{W}\right)
$$

The goal of any optimization is to achieve the maximum values of performance indicators while meeting the specified limits. At the stage of setting the problem, the researcher determines a set of parameters that need to be maximized, minimized, or limited. The indicators are optimized by varying the input parameters. The researcher sets the composition of the variable variables, the ranges of their changes, and, possibly, the relationship with other input parameters.

Usually, when conducting optimization studies, it is necessary to solve not one, but several optimization problems that differ:

- the composition of variable variables (from minimal changes in the project to a complete redesign);

- values of constraints (analysis of the possibility of relaxing individual requirements for the project);

- the number and composition of optimization criteria (from a complete set of alternative projects to a single option).

In the course of solving the problem, it is necessary to solve the problem of finding the maximum of the objective function $f(x)$ :

$$
\max _{\boldsymbol{x}} f(\boldsymbol{x}) \triangleq \sum_{i=1}^{N} P_{i}\left(\boldsymbol{\alpha}, \boldsymbol{o}, U, \theta^{W}\right), \quad \boldsymbol{x}^{l} \leq \boldsymbol{x} \leq \boldsymbol{x}^{u},
$$

where $\boldsymbol{x}=\left(\alpha_{1}, o_{1}, \ldots, \alpha_{N}, o_{N}\right), N$ - the number of wind turbines.

The generated power of wind turbines depends on 4 parameters: the angle of rotation of the blades (angle of attack), the values of the flow velocity, the angle of direction of the vector flow velocity, yaw rate. The range of changes in 2 selected parameters out of 4 set according to changes in weather data in the area of the location of the wind farm in the town Tiksi, as well as the technical characteristics of a wind turbine with a power of $P=0.3 \mathrm{MW}$. The range for changing all parameters is known, the yaw angle $\boldsymbol{o}$ varies from $0^{\circ}$ to $25^{\circ}$, the angle of rotation of the blade $\alpha$ from $-10^{\circ}$ to $10^{\circ}$.

\section{Analytical Models for Calculation of Wake Parameters}

Analytical models in comparison with models based on the solution of partial differential equations are simpler and require less computing resources of the computer. The open package FLORIS (FLOw Redirection and Induction in Steady-state) contains various analytical models for calculating the vortex traces 
of wind turbines in the wind farm, [18]. The FLORIS package was developed in the Python programming language at NREL (USA), TU Delft.

One of the first analytical models for calculating the parameters of the vortex wake was developed by N. Jensen in Denmark in 1983 [19]. In this paper, it was proposed to use the "top-hat shape" model for the cylinder to calculate the velocity deficit in the track (see Fig. 8) and written in the form:

$$
\frac{\Delta U}{U_{\infty}}=\left(1-\sqrt{1-c_{T}}\right) /\left(1+\frac{2 k_{\text {wake }} x}{d_{0}}\right)^{2},
$$

where $\frac{\Delta U}{U_{\infty}}$ is a normalized (dimensionless) velocity deficit.

$$
\frac{\Delta U}{U_{\infty}}=\frac{U_{\infty}-U_{W}}{U_{\infty}}
$$

The Eq. (19) has been widely used in the literature and has been implemented in commercial software (WasP, WindPRO, WindSim). However, there were two limitations to this model: 1) the assumption of the distribution of the velocity deficit was unrealistic; 2) only the law of conservation of mass was used to derive Eq. (19).

(a)

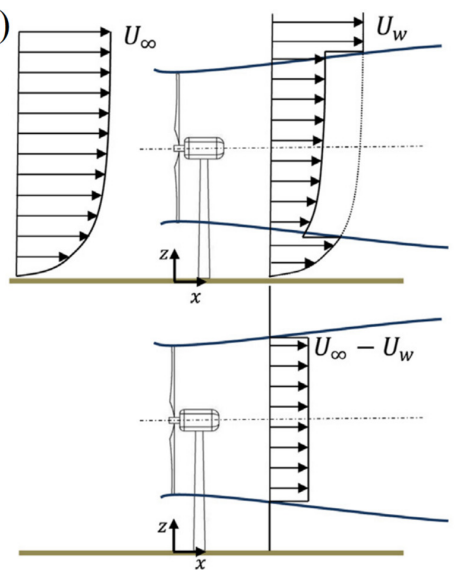

(b)

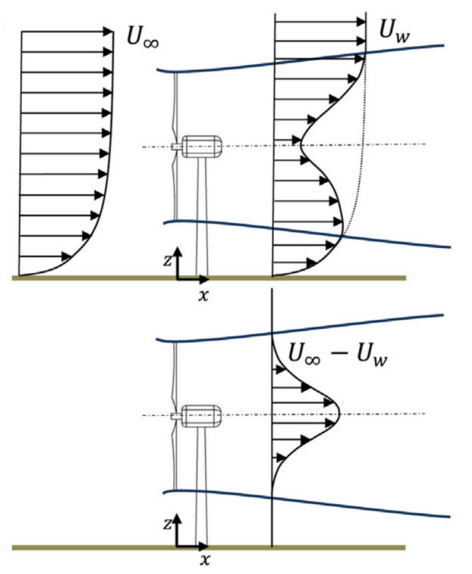

Fig. 8. Velocity profile for the wind turbine according to the a) "top-hat shape" model; b) Gaussian distribution model. Picture is taken from [20].

Subsequently, Frandsen [21] applied the equations of conservation of mass and amount of motion for the control volume (Fig. 9) around the wind turbine and proposed the following expression:

$$
\frac{\Delta U}{U_{\infty}}=\frac{1}{2}\left(1-\sqrt{1-2 \frac{A_{0}}{A_{W}} c_{T}}\right) .
$$




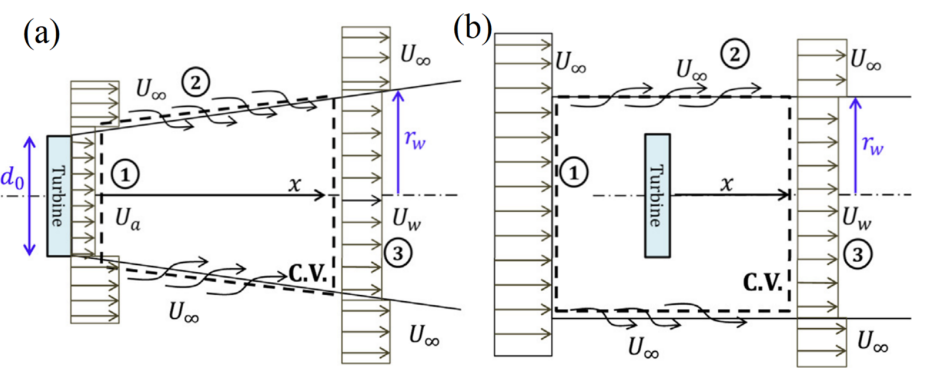

Fig. 9. Example of using the control volume: a) quadrilateral; b) rectangle. Picture is taken from [20].

Vortex traces behind bodies in free flow have been actively studied in the theory of shear flows. In these studies, a self-similar Gaussian profile was found to calculate the velocity deficit.

The self-similar Gauss profile was discovered in experiments in wind tunnels, in numerical calculations, and in operating wind farms. An analytical model with a self-similar Gauss profile was obtained in [20]:

$$
\begin{aligned}
\frac{\Delta U}{U_{\infty}}= & \left(1-\sqrt{1-\frac{c_{T}}{8\left(k^{*} x / d_{0}+\varepsilon\right)^{2}}}\right) \\
& \times \exp \left(-\frac{1}{2\left(k^{*} x / d_{0}+\varepsilon\right)^{2}}\left\{\left(\frac{z-z_{h}}{d_{0}}\right)^{2}+\left(\frac{y}{d_{0}}\right)^{2}\right\}\right) .
\end{aligned}
$$

The analytical models (19)-(22), which were proposed by different authors to predict the deficit of the streamwise velocity in the of wind turbine, were implemented in open source software code FLORIS. FLORIS code is written on Python and is available on github.com [18].

In this paper, the calculation was carried out for a model wind farm with 3 wind turbines according to the Jimenez model, taking into account the influence of changes in the yaw angle for the nacelle [7].

An example of the calculation for a wind farm in the FLORIS package is shown in Fig. 10. The size of the numerical domain was selected with $500 \mathrm{~m}$ in the OY direction and $3200 \mathrm{~m}$ in the OX direction. The velocity was defined as $8 \mathrm{~m} / \mathrm{s}$ at the inlet of the numerical domain. The wind farm consisted of 3 NREL $5 \mathrm{MW}$ wind turbines. They were located on the same straight line, respectively, with coordinates $0,7 \mathrm{D}, 14 \mathrm{D}$.

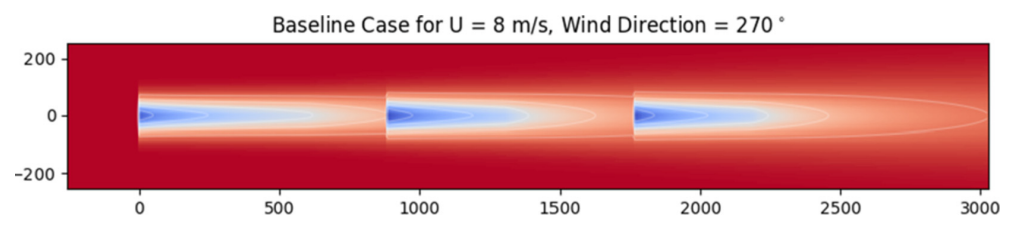

Fig. 10. Initial position of wind turbines in wind farm. 


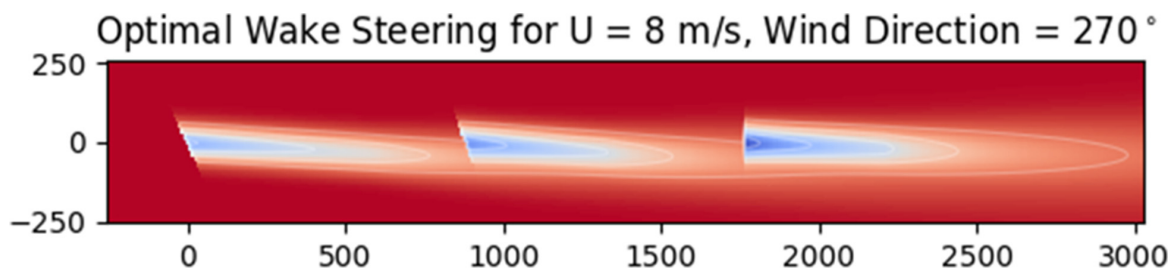

Fig. 11. Velocity value field for 3 wind turbines.

The wind direction was $270^{\circ}$. The initial position of wind turbines is shown in Fig. 10.

The possible range of yaw angle changes for wind turbines is from 0 to $25^{\circ}$. We used the SLSQP (Sequential Least SQuares Programming) optimization algorithm. SLSQP is a nonlinear, gradient-based algorithm that can handle inequality constraints. Iterations are generated by solving quadratic sub-problems. The solution to the optimization problem, the best option is to rotate the nacelle for the first wind turbine is at a 25-degree angle, for the 2nd wind turbine the yaw angle is $19^{\circ}$, for the 3 rd wind turbine the yaw angle is $4.3^{\circ}$ (Fig. 11). The total power gain amounted to $15.1 \%$.

On of the future directions of work could be dealt with using Globalizer Software, developed at the Lobachevsky National Research University for multiextreme optimization problems, see the paper [22] to solve the optimization problems for wind farms.

\section{Conclusion}

This approach, using meteorological data, wind farm data, open-source software WRF-ARW and FLORIS, allows us to formulate the problem of choosing the optimal parameters for the operation of wind turbines in the wind farm. In the future, it is planned to apply the chosen approach to solve the problem of choosing the optimal parameters for the operation of 3 Japanese wind turbines in the wind farm in the Arctic town Tiksi, taking into account the wind rose and complex terrain.

\section{References}

1. Skamarock, W.C., et al.: A description of the advanced research WRF model version 4. UCAR/NCAR (2019). https://doi.org/10.5065/1DFH-6P97

2. Jiménez, P.A., Navarro, J., Palomares, A.M., Dudhia, J.: Mesoscale modeling of offshore wind turbine wakes at the wind farm resolving scale: a composite-based analysis with the weather research and forecasting model over horns rev. Wind Energy 18(3), 559-566 (2014). https://doi.org/10.1002/we.1708 
3. Fitch, A.C., et al.: Local and mesoscale impacts of wind farms as parameterized in a mesoscale NWP model. Mon. Weather Rev. 140(9), 3017-3038 (2012). https:// doi.org/10.1175/mwr-d-11-00352.1

4. Ivanov, A., Strijhak, S., Zakharov, M.: Modeling weather conditions in the port area and coastal zone of Tiksi bay. Proc. Inst. Syst. Program. RAS 31(6), 163-176 (2019). https://doi.org/10.15514/ispras-2019-31(6)-9

5. González, J.S., Rodríguez, A.G., Mora, J.C., Burgos Payán, M., Santos, J.R.: Overall design optimization of wind farms. Renew. Energy 36(7), 1973-1982 (2011). https://doi.org/10.1016/j.renene.2010.10.034

6. Meyers, J., Meneveau, C.: Optimal turbine spacing in fully developed wind farm boundary layers. Wind Energy 15(2), 305-317 (2011). https://doi.org/10.1002/ we. 469

7. Gebraad, P.M.O., et al.: Wind plant power optimization through yaw control using a parametric model for wake effects-a CFD simulation study. Wind Energy 19(1), 95-114 (2014). https://doi.org/10.1002/we.1822

8. Goit, J.P., Meyers, J.: Optimal control of energy extraction in wind-farm boundary layers. J. Fluid Mech. 768, 5-50 (2015). https://doi.org/10.1017/jfm.2015.70

9. Bokharaie, V.S., Bauweraerts, P., Meyers, J.: Wind-farm layout optimisation using a hybrid Jensen-LES approach. Wind Energy Sci. 1(2), 311-325 (2016). https:// doi.org/10.5194/wes-1-311-2016

10. Park, J., Law, K.H.: A data-driven, cooperative wind farm control to maximize the total power production. Appl. Energy 165, 151-165 (2016). https://doi.org/ 10.1016/j.apenergy.2015.11.064

11. Park, J., Law, K.H.: Bayesian ascent: a data-driven optimization scheme for realtime control with application to wind farm power maximization. IEEE Trans. Control Syst. Technol. 24(5), 1655-1668 (2016). https://doi.org/10.1109/tcst.2015. 2508007

12. Hou, P., Hu, W., Soltani, M., Chen, C., Chen, Z.: Combined optimization for offshore wind turbine micro siting. Appl. Energy 189, 271-282 (2017). https:// doi.org/10.1016/j.apenergy.2016.11.083

13. Hou, P., Hu, W., Soltani, M., Chen, Z.: Optimized placement of wind turbines in large-scale offshore wind farm using particle swarm optimization algorithm. IEEE Trans. Sustain. Energy 6(4), 1272-1282 (2015). https://doi.org/10.1109/tste.2015. 2429912

14. Bastankhah, M., Porté-Agel, F.: Wind farm power optimization via yaw angle control: A wind tunnel study. J. Renew. Sustain. Energy 11(2) (2019). https:// doi.org/10.1063/1.5077038

15. Bastankhah, M., Porté-Agel, F.: Experimental and theoretical study of wind turbine wakes in yawed conditions. J. Fluid Mech. 806, 506-541 (2016). https://doi. org $/ 10.1017 / \mathrm{jfm} .2016 .595$

16. Fleming, P.A., Ning, A., Gebraad, P.M.O., Dykes, K.: Wind plant system engineering through optimization of layout and yaw control. Wind Energy 19(2), 329-344 (2015). https://doi.org/10.1002/we.1836

17. Astolfi, D., Castellani, F., Natili, F.: Wind turbine yaw control optimization and its impact on performance. Machines 7(2), 41 (2019). https://doi.org/10.3390/ machines7020041

18. NREL: FLORIS. Version 2.2.3 (2020). https://github.com/NREL/floris

19. Jensen, N.: A note on wind generator interaction. No. 2411 in Ris $\varnothing-M$, Ris $\varnothing$ National Laboratory (1983) 
20. Bastankhah, M., Porté-Agel, F.: A new analytical model for wind-turbine wakes. Renew. Energy 70, 116-123 (2014). https://doi.org/10.1016/j.renene.2014.01.002

21. Frandsen, S., et al.: Analytical modelling of wind speed deficit in large offshore wind farms. Wind Energy 9(1-2), 39-53 (2006). https://doi.org/10.1002/we.189

22. Gergel, V., Barkalov, K., Sysoyev, A.: Globalizer: a novel supercomputer software system for solving time-consuming global optimization problems. Numer. Algebra Control Optim. 8(1), 47-62 (2018). https://doi.org/10.3934/naco.2018003 